\begin{abstract}
We prospectively studied the incidence of methicillin-resistant Staphylococcus aureus (MRSA) colonisation and infection, the patterns and types of operation associated with MRSA acquisition in an orthopaedic and trauma unit in London, UK. Over the 12-month study period from January to December 2000, we found that $1.6 \%$ of the total admission was diagnosed to be either MRSA infected or colonised, with an average of three new MRSA cases detected per month. A significant proportion of patients $(23 \%)$ were diagnosed within the first $48 \mathrm{~h}$ of admission. Both hip joint surgery, especially emergency procedures for femoral neck fractures, and the presence of a wound presented higher risk of infection. The Intensive Care Unit (ICU) did not appear to be a significant source for intra-hospital dissemination among the orthopaedic patients. MRSA infection or colonisation contributed to an increased length of hospital stay; 88 days compared to 11 days on average for nonMRSA patients; $41 \%$ of the positive patients still carried MRSA on discharge. Our data show the importance of diagnosing MRSA in orthopaedic surgery and emphasises that understanding its epidemiology will be crucial to secure a decrease in the incidence of MRSA. Hand hygiene, patient screening, careful surveillance of infections and the prompt implementation of isolation policies, are essential components of control.
\end{abstract}

Résumé Nous avons étudié de façon prospective la fréquence de colonisation et d'infection par le SAMR, les

C. C. Tai · A. A. Nirvani · S. P. F. Hughes Department of Musculoskeletal Trauma,

Imperial College School of Medicine, Charing Cross Campus, London, UK

\section{A. Holmes}

Department of Infectious Disease \& Microbiology,

Imperial College School of Medicine,

Charing Cross Campus, London, UK

C. C. Tai ( $)$

45 Achilles Road, West Hampstead, London, NW6 1DZ, UK e-mail: cctai@doctors.net.uk

Tel.: +44-20-77949246, Fax: +44-20-77949246 modèles et types d'opération associés avec l'acquisition de SAMR dans une unité d'orthopédie et traumatologie à Londres. Sur la période de l'étude, douze mois de janvier à décembre 2000, nous avons trouvé que 1,6\% des admissions portaient le diagnostic de colonisation ou d'infection à SAMR, avec une moyenne de trois nouveaux cas détectés par mois. Une proportion importante de malades $(23 \%)$ a été diagnostiqué dans les 48 premières heures d'admission. La chirurgie de la hanche, surtout les traitements en urgence pour fracture du col fémoral, et la présence d'une blessure avaient des risques supérieurs d'infection. L'Unité de soins intensifs n'est pas apparue comme une source significative de dissémination intra-hospitalière parmi les malades orthopédiques. L'infection ou la colonisations à SAMR ont contribué à un allongement du séjour à l'hôpital : 88 jours, comparés à 11 jours en moyenne pour les malades sans SAMR. Nos données montrent l'importance de diagnostiquer la présence de SAMR en chirurgie orthopédique et de comprendre son épidémiologie pour assurer une décroissance de l'incidence des infections à ce type de germe. L'hygiène des mains, l'étude des patients, la surveillance soigneuse des infections et la mise en oeuvre rapide de politiques d'isolement, sont des composantes essentielles du contrôle.

\section{Introduction}

The isolation of methicillin-resistant strains of Staphylococcus aureus (MRSA) from clinical sources was first described in 1961 [9]. Initial reports were usually of sporadic isolates, but by the late 1970s, epidemic outbreaks were increasingly described in hospitals all over the world $[6,12]$. Up to $30 \%$ of $S$. aureus strains isolated from nosocomial infections are MRSA [14], and it has become a growing problem in high-risk units, such as orthopaedic departments, where patients undergo implant surgery. Much has been written about MRSA outbreaks in hospitals and the general community [19] and also about the management and control of patients infected or 
colonised with MRSA. Other studies have investigated the MRSA infection rates in various parts of the world. A study conducted in the surgical department in Japan in 1993 showed that MRSA constituted 3\% of all infections [20]. Studies on nursing homes in the United States [4, 22] found that the monthly bacterial colonisation rate ranged from $6.6 \%$ to $23 \%$, with an MRSA infection rate of $3 \%$. In Europe, the reported MRSA infection rates varies widely, from less than $3 \%$ reported in Scandinavia and Germany to greater than $30 \%$ in Spain, France and Italy $[3,23]$.

In this paper, we have investigated the incidence of MRSA infection and colonisation over a 1-year period in the orthopaedic and trauma department in a teaching hospital in London. In addition, both the patterns of MRSA acquisition and the risk factors associated with the types of operation within the department were assessed.

\section{Patients and methods}

The study was conducted in the Department of Orthopaedic Surgery at a tertiary-care teaching hospital in the north-west Thames region in London. The department is located on a single floor and is divided into three geographically separate wards comprising 73 beds with a total of six single rooms in the three wards. One ward functions on a 5-day basis. There is no separation of elective and emergency patients. All nursing staff carries out their primary duties within the confines of a single ward, but there is extensive movement of doctors and other health care professionals between the wards.

All high risk patients, i.e. patients previously documented as MRSA carriers or those who have been transferred from other institutions, were screened on admission for MRSA colonisation.

The prospective study was conducted over a 12 -month period between January and December 2000. The following information of patients colonised or infected with MRSA was collated: age, gender, date of and reasons for admission, patient placement prior to admission and subsequent to discharge, duration of stay, type of operations performed, time interval between admission/operation and diagnosis of colonisation/infection, and the MRSA status on discharge. MRSA infected or colonised inpatients diagnosed prior to 1 January 2000, or new MRSA-positive patients diagnosed in the outpatient clinics between 1 January and 31 December 2000 were excluded from this study.

The specimens screened comprised cotton-tipped swabs used for bacteriological investigation of the nose, throat, axilllae and groin. In addition, swabs from skin lesions and wounds, if any, were also cultured. Urine, blood cultures and sputum specimens were analysed where clinically indicated and were defined as clinical specimens. When new MRSA cases were diagnosed, all patients were isolated within $24 \mathrm{~h}$ and the standard MRSA protocol [18] was implemented immediately. Treatment was discontinued when three consecutive sets of cultures did not yield organisms.

Colonisation was defined as one or more MRSA-positive cultures from any screen sites without the accompanying classical signs and symptoms of infection. Infections were defined based on both clinical and microbiological data. A successful clinical outcome was defined by resolution of the signs and symptoms of infection with concomitant elimination of MRSA from the previous positive sites.
Table 1 Source and type of admission of methicillin-resistant Staphylococcus aureus- (MRSA) positive cases. Four patients were transferred from other hospitals, two of them were from other regions. Two patients were intra-hospital transfer, one from the medical ward and the other from a geriatric ward within the same building but from different floors

\begin{tabular}{llll}
\hline $\begin{array}{l}\text { Admission } \\
\text { from }\end{array}$ & $\begin{array}{l}\text { Number of } \\
\text { patients }\end{array}$ & $\begin{array}{l}\text { Admission } \\
\text { type }\end{array}$ & $\begin{array}{l}\text { Number of } \\
\text { patients }\end{array}$ \\
\hline Home & 25 & Emergency & 19 \\
Transfer & 6 & Elective & 12 \\
Total & 31 & Total & 31 \\
\hline
\end{tabular}

Table 2 Type of clinical specimen and the number of methicillinresistant Staphylococcus aureus- (MRSA) infected patients treated with intravenous antibiotics. None of the MRSA-positive cases from screen specimen required intravenous antibiotics. ${ }^{\mathrm{a} O n e}$ patient was diagnosed to be MRSA positive from both wound and blood cultures

\begin{tabular}{lll}
\hline $\begin{array}{l}\text { Clinical } \\
\text { Specimen }\end{array}$ & $\begin{array}{l}\text { Number of } \\
\text { patients }\end{array}$ & $\begin{array}{l}\text { Patients treated with } \\
\text { i.v. antibiotics }\end{array}$ \\
\hline Wound & $14^{\mathrm{a}}$ & 7 \\
External pin site & 2 & 0 \\
Blood & 2 & 2 \\
Sputum & 2 & 2 \\
Urine & 1 & 0 \\
Total & 21 & 11 \\
\hline
\end{tabular}

\section{Results}

A total of 1,879 patients, inclusive of both elective and emergency cases, were admitted over the 12-month period. During this period, 121 patients were screened and 1,144 screen specimens were collected. Of these, 31 new cases (colonised and infected) were detected (Table 1), 21 were diagnosed based on clinical specimens (Table 2) and ten on screening specimens. The monthly incidence of MRSA cases was on average 2.6 cases. These comprised 18 women and 13 men ranging in age from 19 to 90 years, with the mean age 65 years. None of the six inter- or intra-hospital referral patients found to be MRSA positive were known to be infected or colonised with MRSA prior to transfer.

Notably, a significant proportion of patients (seven out of 31) were diagnosed within the first $48 \mathrm{~h}$ of admission. The mean time from admission to first positive culture was $27.4 \pm 6.2$ days. Twenty-six patients underwent operations, and the mean time from operation to first positive culture was $26.5 \pm 6.1$ days. The length of hospital stay was an average of 13 weeks. Two patients were still in-house at the completion of the study and two patients died of causes unrelated to their MRSA infection.

Twenty-five MRSA-infected patients were found in the three orthopaedic wards: there were 11 and ten cases, respectively, in two full-time orthopaedic wards and four patients in the five-day orthopaedic ward. Three other orthopaedic patients were distributed in three different 
general surgical and vascular surgical wards on different levels of the hospital. Two were in private wards on different floors from the orthopaedic wards. One was diagnosed as MRSA positive while she was in the Intensive Care Unit (ICU), which is on a different floor. A total of nine and 15 patients were operated on in the two main orthopaedic theatres, respectively, whereas two patients were operated on in a separate emergency theatre.

All colonised and infected patients were treated with standard MRSA protocols [11] but antibiotics treatment was prescribed to only 11 infected patients. No patient colonised with MRSA on screening subsequently developed infection.

On discharge, 11 out of 27 patients still carried MRSA. With two exceptions, all 13 patients who were MRSA positive from their surgical wounds were clear prior to discharge. None of these patients had open wounds, and all the wounds healed before discharge. Twenty-four patients were discharged home; three were transferred to a nearby rehabilitation centre and all three were clear of MRSA colonisation or infection prior to transfer.

\section{Discussion}

We found an average of three new MRSA colonised or infected patients detected per month, representing $1.6 \%$ of the total admissions over the 12-month period. The incidence is lower than that reported from hospitals in other European countries [3, 23], but similar to the figures from the McNeil survey (1.8\%), which studied the prevalence of MRSA in all wards across specialities in an Australian teaching hospital [13]. This may be due, at least in part, to the active infection-control program at this hospital to monitor the presence of MRSA and to prevent its spread. The new colonised or infected cases did not show any striking pattern insofar as wards or operating theatres. Surprisingly, although the ICUs were previously reported to be a significance source for intrahospital dissemination of MRSA [11], only one orthopaedic case was found to be MRSA positive from the ICU.

The interval between admission and positive culture ranged from 1 to 92 (mean 27) days, in contrast to data from a community hospital in the United States that ranged from 4 to 56 days [10]. Interestingly, more than a third of our patients were diagnosed MRSA positive within the first week of their admission. In fact, a remarkably high proportion (7/31) was diagnosed within the first $48 \mathrm{~h}$, suggesting that these patients were colonised or infected prior to admission. Similar figures were also observed in the McNeil study in Australia [13]. This includes two of the four inter-hospital-transferred patients who were found to be MRSA positive on the first day of their admission, despite no confirmation of MRSA infection or colonisation prior to transfer. Since one of the main sources of transmission of MRSA between hospitals is indeed via the transfer of patients [11], it is imperative to screen transferred patients on arrival and to establish good inter-hospital communications.

Twenty-six MRSA-positive patients in our study had undergone surgery. Twenty-four patients were diagnosed post-operatively, with half diagnosed within the first 2 weeks. This represents an incidence of MRSA colonisation and infection of less than $1 \%$ (26 of 1,844 operations) over the 12-month period. More significantly, the incidence of MRSA infection and colonisation is significantly higher in patients who have undergone operative procedures on the hip joints. In this hospital, $14 \%$ of the orthopaedic operations were for hip-joint operations and yet $65 \%$ of the MRSA cases had hip-joint procedures. These results are consistent with those in Germany, where hip operations have the highest risk for MRSA infection and the symptoms start 4-7 days after surgery [23]. Emergency hip operations in particular present a higher risk - in our study population, $16 \%$ of total emergency hip cases were subsequently infected or colonised in comparison to only $3 \%$ of total elective hip operations. Emergency operations involving external fixation performed on the tibia also appeared to carry relatively high risk, with $5 \%$ of the total emergency tibial operations found to be MRSA positive. Most of the colonisation was related to the pin sites of the external fixation. On the other hand, less than $1 \%$ of the patients with total knee operations, all of which were elective, were MRSA positive. Ankle and spinal operations presented relatively low risk of infection. Interestingly, all the MRSA infected or colonised cases in both ankle and spinal operations were emergency procedures. Other factors that might contribute to increased risk of infection include age (mean age 65) and the presence of a wound.

The mean length of stay for MRSA-negative patients was 11 days whereas the mean length for MRSA-positive patients was 88 days. Extended stay of MRSA patients (average 74 days) was also reported in a study conducted at a 700-bed hospital in the United States [15]. In our study, the mean length of stay before MRSA infection was 27 days, and therefore the increased stay of MRSA patients was probably due to infection. The increased length of stay undoubtedly has significant impact on the cost, time and labour in the National Health Service (NHS) in providing care to these patients.

In our study, 11 patients required intravenous antibiotics. This represents more than $50 \%$ of the patients who were diagnosed MRSA positive from clinical specimens. In contrast, none of the positive cases from screen specimens required intravenous antibiotics. It is noteworthy that we did not find any other patients who were infected and required antibiotic treatment following colonisation.

The discharge of MRSA-colonised patients to the community has contributed to the growing problem of nosocomial MRSA infection. MRSA colonisation is notoriously persistent as it can be diagnosed after 4 weeks of treatment from $46 \%$ of colonised individuals [8] and up to 2-3 years after discharge [5]. Persistent carriers therefore pose a risk. Our policy is only to screen patients considered as high risk or who are known carriers; 
however, patients may move around, especially in large cities such as London, which make detection and followup particular difficult. On the other hand, it is perhaps equally important to point out that both German and Australian studies have shown that early discharge of MRSA-colonised patients to home can effectively contribute to reduce the intra-hospital spread of MRSA [16, 23].

This study has shown that both the MRSA infection and colonisation rates are low. In addition, patients are unlikely to develop MRSA infection following colonisation. Therefore, the cost effectiveness of aggressive management of MRSA (widespread screening for MRSA and eradication with extensive antimicrobial agents) may need to be assessed. There are other cheaper and perhaps more productive options. By far the most effective preventive measure is to educate doctors and nurses regarding their role as vectors. The notion that MRSA carriers have poor colonising potential no longer holds true $[2,7$, 11]. The transmission of MRSA by transiently colonised or contaminated hands of the personnel is regarded as the main route [15]. Simple measures, such as the role of meticulous hand hygiene, in infection control is well established $[17,21]$ and needs to be re-emphasised. Contamination of the patient's environment (e.g. medical equipment, hospital furnishings, linens) may not directly be a source of infection but may contaminate the hands of hospital personnel. This is perhaps one of the most likely explanations for the failure to stem the spread of MRSA despite the common practice of single-room isolation or cohorting for MRSA patients [1].

In recent years, there has been an increasing recognition of the importance of surveillance of hospitalacquired infection in the United Kingdom. Hospitalacquired infection causes significant morbidity and mortality and cost the NHS an estimated $£ 1$ billion per year. It has been estimated that one third of infections are preventable. The first step in MRSA infection control is the identification of the major MRSA reservoir-i.e. both colonised and infected patients. Unfortunately, the current data on MRSA colonisation and infection in the United Kingdom is scarce, and hence the impact of MRSA on NHS is largely undetermined. We would therefore advocate regular and careful surveillance of MRSA infections, not only in orthopaedics but also in other high-risk departments. This tremendous task should not be shouldered solely by the infection control team in the hospital but is the responsibility of every health care provider. Orthopaedic surgeons, in particularly, must take a leading role in understanding and preventing cross transmission of MRSA in light of its increasing threat to the field of orthopaedics.

\section{References}

1. Barakate MS, Yang Y-X, Foo S-H, et al (2000) An epidemiological survey of methicillin-resistant Staphylococcus aureus in a tertiary referral hospital. J Hosp Infect 44:19-26
2. Bartzokas CA, Paton JH, Gibson MF et al (1984) Control and eradication of methicillin resistant Staphylococcus aureus on a surgical unit. N Eng J Med 29:1422-1425

3. Beaujean DJ, Weersink AJ, Blok HE et al (1999) Determining risk factors for methicillin-resistant Staphylococcus aureus carriage after discharge from hospital. J Hosp Infect 42:213-218

4. Bradley SF, Terpenning MS, Ramsey BS et al (1991) Methicillin-resistant Staphylococcus aureus: colonization and infection in a long term care facility. Ann Intern Med 115:417-422

5. Frenay HM, Vandenbroucke-Grauls CM, Molkenboer MJ et al (1992) Long-term carriage, and transmission of methicillinresistant Staphylococcus aureus after discharge from hospital. J Hosp Infect 22:207-115

6. Haley RW, Hightower AW, Khabbaz RF et al (1982) The emergence of methicillin-resistant Staphylococcus aureus infection in United States hospitals. Ann Int Med 97:297-308

7. Hancox R, Cummins A, Kelsey MC (1992) An outbreak of MRSA associated with long term colonization of medical staff. J Hosp Infect 22:170-172

8. Hicks NR, Moore EP, Williams EW (1991) Carriage and community treatment of methicillin-resistant Staphylococcus aureus: What happens to colonised patients after discharge? J Hosp Infect 19:17-24

9. Jevons MP (1961) "Celbenin"-resistant staphylococci. Br Med J 1:124-125

10. Klimek JJ, Marsik FJ, Bartlett RC et al (1976) Clinical, epidemiologic and bacteriologic observations of an outbreak of methicillin-resistant Staphylococcus aureus at a large community hospital. Am J Med 61:340-345

11. Locksley RM, Cohen ML, Quirm TC et al (1986) Multiply antibiotic-resistant Staphylococcus aureus: introduction, transmission, and evolution of nosocomial infection. Ann Intern Med 93:526-532

12. McDonald PJ (1982) Methicillin-resistant staphylococci: a sign of the times? Med J Austr 1:445-446

13. McNeil JJ, Proudfoot AD, Tosolina, FA et al (1984) Methicillin-resistant Staphylococcus aureus in an Australian teaching hospital. J Hosp Infect 5:18-28

14. Paul-Ehrlich-Gesellschaft fur Chemotherapie (1990) European study of bacterial resistance. Report Chapter IV. part J

15. Peacock JEJr., Marsik FJ, Wezel RP (1980) Methicillin-resistant Staphylococcus aureus: introduction and spread within hospital. Ann Intern Med 93:526-532

16. Pearman JW, Christiansen J, Annear DL, et al (1985) Control of methicillin-resistant Staphylococcus aureus (MRSA) in an Australian metropolitan teaching hospital complex. Med J Aust 142:103-108

17. Pittet D, Hugonnet S, Harbarth $S$ et al (2000) Effectiveness of a hospital-wide programme to improve compliance with hand hygiene. Infection Control Programme. Lancet 356 9238): $1307-1312$

18. Revised guidelines for the control of epidemic methicillin-resistant Staphylococcus aureus (1998) Report of a combined working party of the hospital Infection Society and British Society for Antimicrobial Chemotherapy. J Hosp Infect 39:253-290

19. Saravolaz LD, Markowitz N, Arking 1 et al (1982) Methicillinresistant Staphylococcus aureus: epidemiologic observations during a community-acquired outbreak. Ann Intern Med 96:11-16

20. Shimada M, Kamakura T, Itasaka H et al (1993) The significance of methicillin-resistant Staphylococcus aureus infection in general surgery: a multivariate analysis of risk factors and preventive approaches. Surgery Today 23:880-884

21. Steere AC, Mallison GF (1975) Handwashing practices for the prevention of nosocomial infections. Ann Intern Med 83:683-690

22. Thomas JC, Bridge J, Waterman S et al (1987) Transmission and control of methicillin-resistant Staphylococcus aureus in a skilled nursing facility. Infect Control 8:24-29

23. Witte W, Braulke C, Heuck D et al (1994) Analysis of nosocomial outbreaks with multiply and methicillin-resistant Staphylococcus aureus (MRSA) in Germany: implications for hospital hygiene. Infection 22 (Suppl) 2:128-134 